\title{
BULLOUS TYPE OF REACTION IN LEPROSY
}

\author{
by DR. C. K. JOB, M.D., \\ Pathologist, Schieffelin Leprosy Research Sanatorium, Karigiri \\ and Dr. G. W. Gault, M.D., M.S., F.R.A.C.S., \\ Professor of Pathology, Christian Medical College, Vellore
}

There are many cases of leprosy with bullous lesions reported in medical literature, though detailed autopsy findings on these cases are not available. Most of these case reports are from workers in South America and it has not been described in patients from India. This is a case report of an Indian patient with lepromatous leprosy who developed the bullous type of lesions and died. The autopsy findings of this case are also described in detail.

\section{REPORT OF A CASE}

A farmer aged 40 years, a native of North Arcot District in South India, was registered in Schieffelin Leprosy Research Sanatorium for the first time on 4th January, 1957. He gave a history of a hypopigmented patch on the right thigh 16 years ago, which gradually increased in size. Six years later, many more hypopigmented, shiny, macular lesions were noticed all over the body for which he had periodical injections of hydnocarpus oil for one year only. The patient's father had leprosy.

Eight months before admission to this hospital, the patient noticed bullous lesions, which after a time, ulcerated and then resolved. He continued to get these lesions at intervals and each episode was worse than the previous one and the ulcers became extensive and deep.

Physical Examination. The general condition of the patient was poor. He was drowsy and no coherent answers could be obtained from him.

The cardiovascular system, the respiratory system and the gastro-intestinal system showed no abnormality. There was bilateral well marked iritis in the eyes. Examination of the Skin. There was generalised diffuse infiltration which involved the entire trunk and face. There were extensive ulcers on both lower and upper extremities. The ulcers were very deep in the legs and feet. The base was red in areas and in places was covered by necrotic tissue. The edges were ragged. Superficial ulcers, involving the cheek and the nose, were seen on the face. There was loss of hair on both eyebrows and thickening of the ears.

Laboratory Data. The significant findings were that his blood haemoglobin was $4.5 \mathrm{~g} . \%$ and his urine showed the presence of albumin, a trace of sugar, many pus cells and occasional granular casts.

Progress. The patient was given Seclopen injections twice a day and blood transfusion of $500 \mathrm{ml}$. of blood. He remained semi-conscious throughout the period of stay in hospital and died after 14 days.

\section{POST MORTEM EXAMINATION}

Post mortem examination was carried out five hours after death.

External Appearance. The body was that of a very emaciated man, weighing $38 \mathrm{~kg}$. and measuring $165 \mathrm{~cm}$. in length. There was diffuse lepromatous infiltration of the skin over the body and face. The eyes were sunken. Both the cheeks, the nose and the ear lobes showed superficial ulceration. In the lower extremities there were extensive deep ulcers with base partly covered with granulation tissue and partly necrotic, and tendons were exposed in some areas. These ulcers involved the entire leg and lower third of the thighs. There were ulcers on the upper extremities also, involving the hand, the forearm, and the lower third of the upper arm. Superficially, the involved areas appeared gangrenous. A few bullous lesions were seen on the extensor aspect of the forearm and the upper arm. The buttocks showed deep ulceration.

Gross Examination:

Liver weighed 1,500 g. It was pale and was increased in size, with round edges. Cut surface showed pale and yellowish appearance. 
Spleen weighed $350 \mathrm{~g}$. It was markedly congested and friable.

Testes. The left testis weighed $18 \mathrm{~g}$. It was firm in consistency. On cut section, no normal testicular tissue was identified, but there was gross evidence of fibrosis.

The right testis showed a small hydrocele containing about $50 \mathrm{ml}$. of strawcoloured fluid. It weighed $20 \mathrm{~g}$. The cut surface showed an appearance similar to that of the left testis.

In the respiratory system, the cardiovascular system, the alimentary system, the urinary system, and the central nervous system, no significant abnormalities were detected.

\section{MICROSCOPIC EXAMINATION}

Sections from all organs were stained with haematoxylin and eosin and for acid fast organisms.

Skin. Pieces of skin were taken from representative areas in the upper and lower extremities. The basic lepromatous picture was seen with flattening of epidermis and a clear area beneath it separating the epidermis from an inflammatory infiltrate consisting of lymphocytes, plasma cells, and many macrophages. The bullae involved the entire thickness of the epidermis. The corium was oedematous, congested, and necrotic in some areas. There were small arteries showing collagenous thickening and panarteritis. The different coats were indistinct and the inflammatory infiltrate was most abundant in the media and the adventitia. The lumina of some of these vessels were very narrow. Small veins and capillaries showed endothelial proliferation and vascularitis. There was also evidence of thrombosis in a few of them.

The subcutaneous fat showed an inflammatory infiltrate predominantly composed of neutrophil granulocytes and also macrophages. This was primarily seen in the septa. The nerve bundles showed fibrosis, and infiltration with lymphocytes and macrophages.

The acid-fast stain showed numerous bacilli inside the macrophages present in the corium and in the subcutaneous tissue. Many bacilli were seen in the interstitial spaces. The endothelial cells of the walls of the blood vessels were distended with acid-fast organisms. The nerve bundles contained many bacilli. Liver. Showed well-marked fatty change of the parenchymal cells. There were small granulomas consisting of lymphocytes, plasma cells, and macrophages situated focally and in the periportal areas. There was slight increase in fibrous tissue but the architecture of the liver was preserved. Many acid-fast bacilli were seen inside macrophages.

Spleen. Showed well marked congestion and scattered granulomas consisting of foamy macrophages, plasma cells and leucocytes. Many acid-fast bacilli were seen inside macrophages in the granulomas and lying free in the sinusoids.

Testes. The seminiferous tubules were atrophic and hyalinized. In the interstitial tissue there were many granulomas consisting of lymphocytes, plasma cells, and macrophages. There was marked increase in fibrous tissue. Acid-fast stain showed many bacilli in the granulomas and in the seminiferous tubules.

The Adrenals. The cortex revealed a few scattered granulomas consisting of plasma cells, leucocytes, and macrophages. But the medulla showed no lesion. Acid-fast stain showed many bacilli packed inside the macrophages.

Microscopically, the heart, the aorta, the lungs, the pancreas, the kidneys, the bladder, the thyroid, the brain, the spinal cord, the pituitary, the gastrointestinal tract showed no abnormality, but the lungs and the kidneys showed acid-fast bacilli in the endothelial cells of the capillaries of the alveolar walls and the glomerular tuft.

\section{DISCUSSION}

Lucio and Alvardo of Mexico (1852) as quoted by Pardo Castello and Pineyro ${ }^{6}$ described a form of leprosy with diffuse lepromatous infiltration, and bullous eruptions which burst giving rise to deep ulceration. They considered it as an entirely new form of the disease and termed it "Lazarine Leprosy".

Pardo Castello and Caballero (1930) ${ }^{5}$ made a study of Lazarine Leprosy and presented it as a peculiar mono-symptomatic form. Their experience was based on 23 Cuban cases. The basic histopathological picture in these cases was thought to be tuberculoid leprosy. 


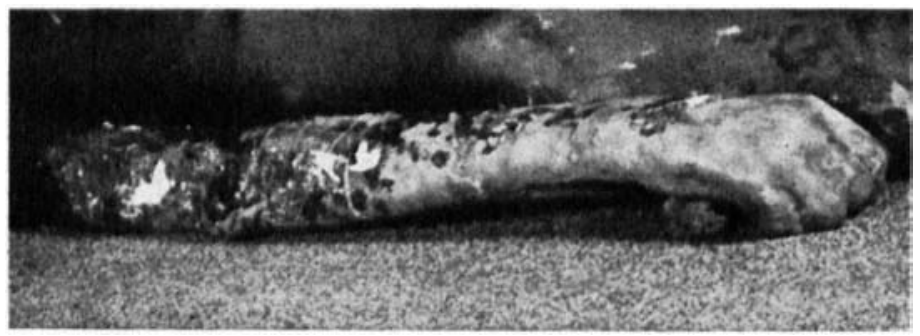

1. The right hand showing extensive ulceration and superficial gangrene.

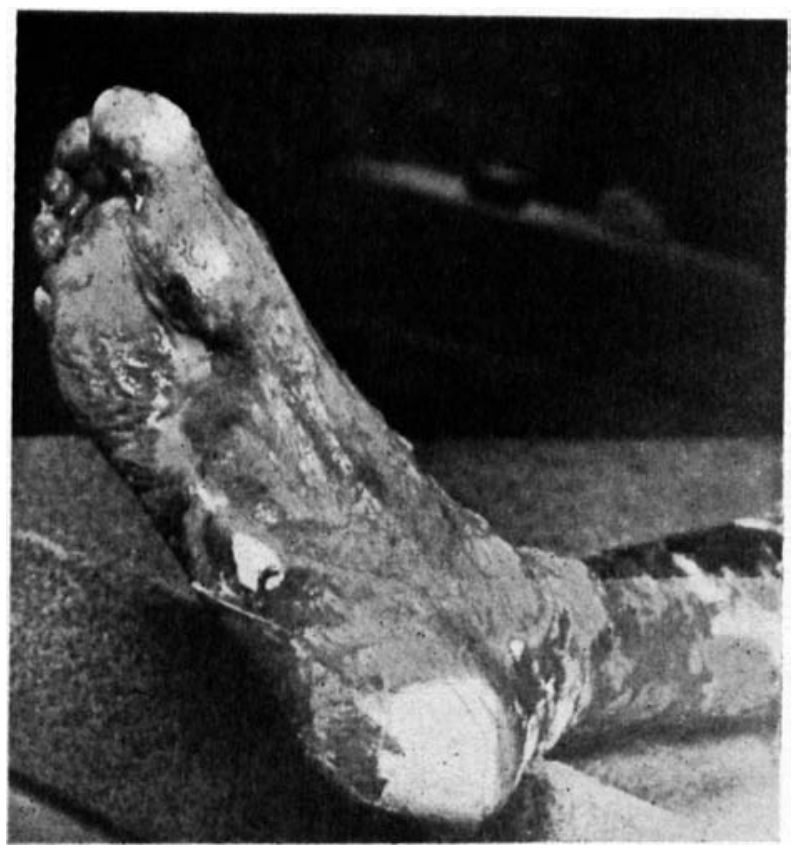

2. The right foot showing extensive deep ulceration. 


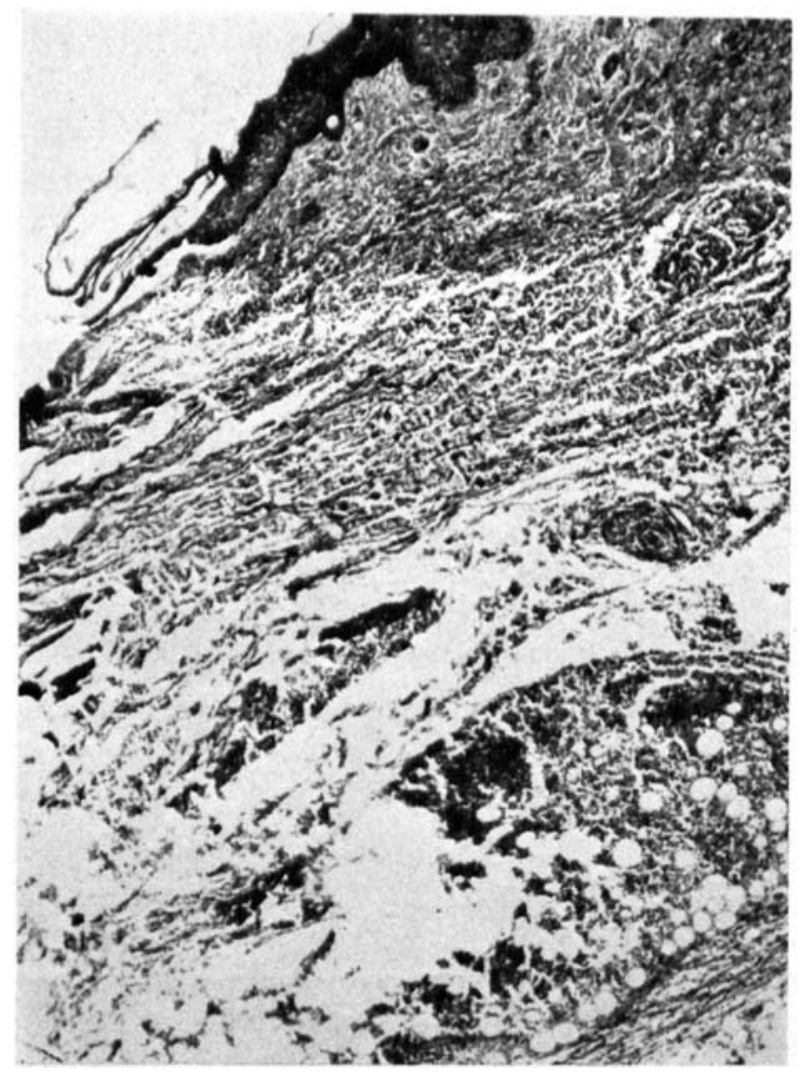

3. Photomicrograph of the skin showing the ulcer with jagged edges aficer the blister has burst. Superficially, there is a large collection of polymorphomuclear leucocytes. Deeper in the dermis there is necrosis and infiltration of fat with polymor phonuclear leucocytes and macrophages.

$H \& E \times 35$.

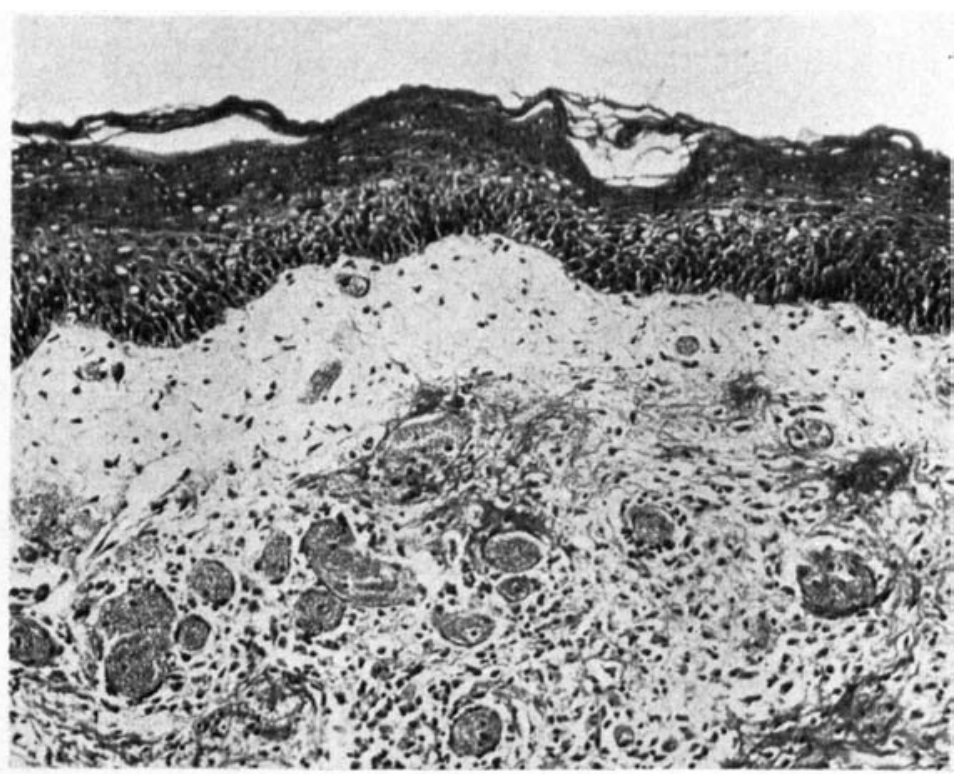

4. Photomicrograph of skin showing marked engorgement of the capillaries adjacent in the hlister area. 


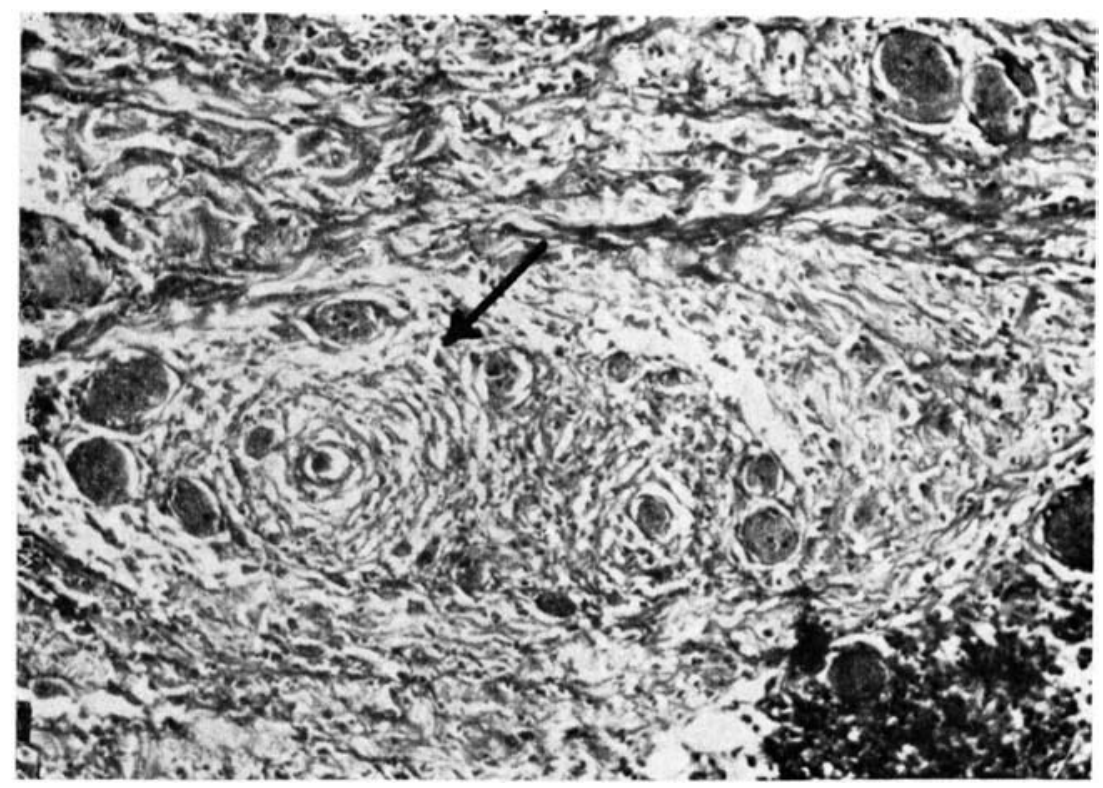

5. Photomicrograph of a small vessel in the deep dermis, showing a necrotizing lesion. The lumen is markedly narrowed and occluded with a thrombus. There is also engorge'me'nt of small blood capillaries around it. $H \& E \times 100$.

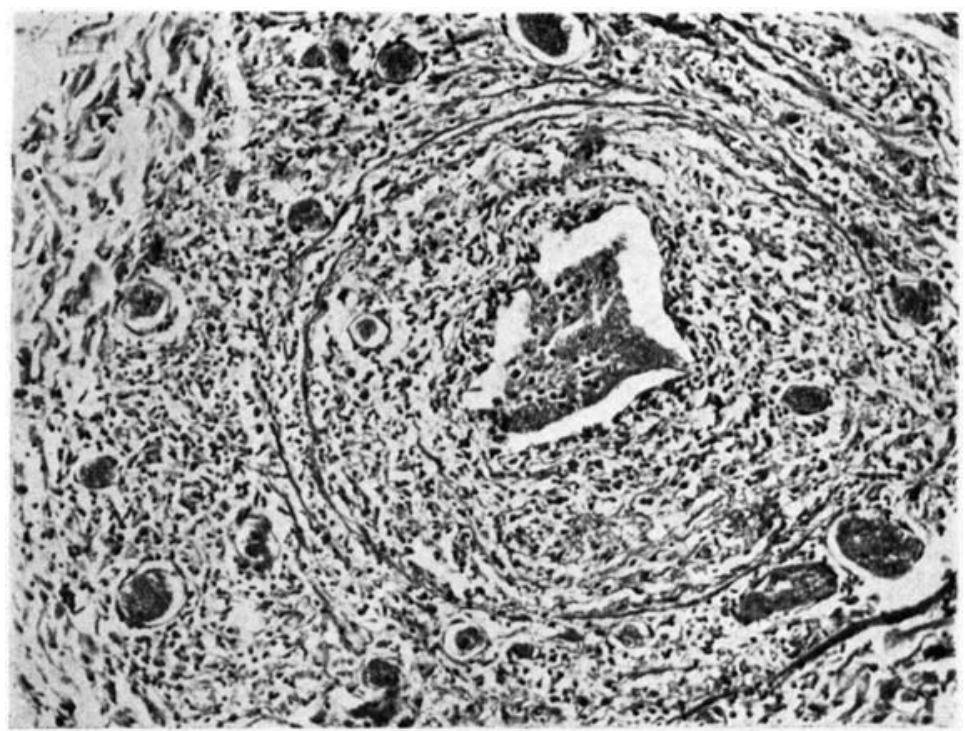

6. Photomicrograph showing a vessel deep in the dermis showing endothelial proliferation, narrowing of the lumen, and infiltration of the entire wall with pol ymor phomuclear leucocytes, lymphocytes and macrophages. There is marked engorgement of the surrounding blood capillaries.

$H \& E \times 100$. 


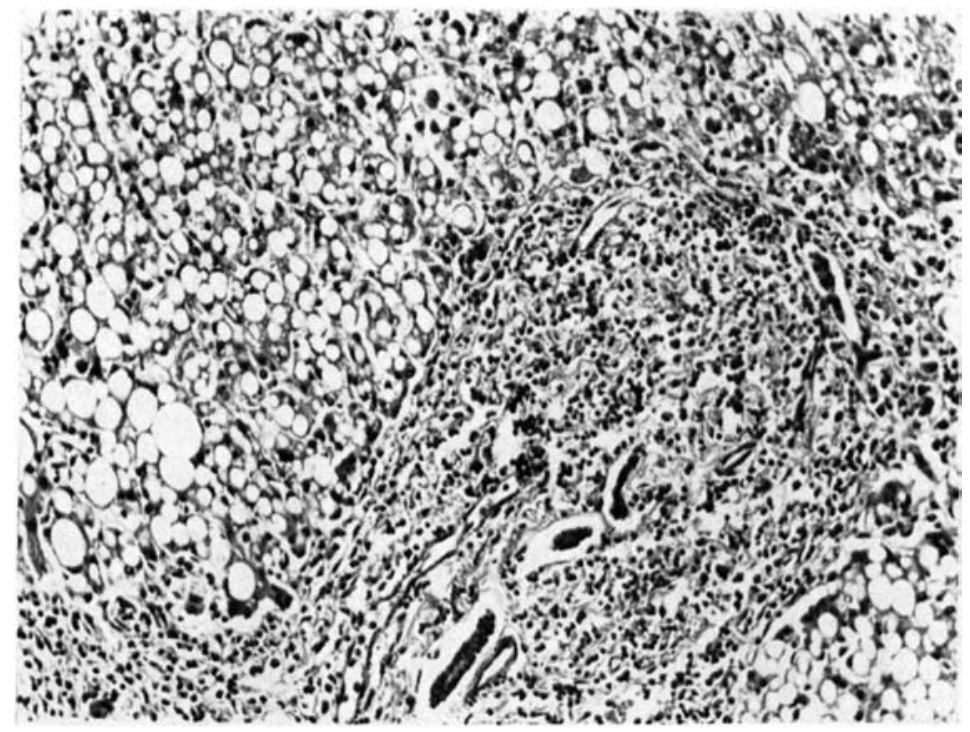

7. Photomicrograph of the liver showing granulomatous inflammation around the portal tract and marked fatty change of the parenchymal cells. $H \& E \times 100$.

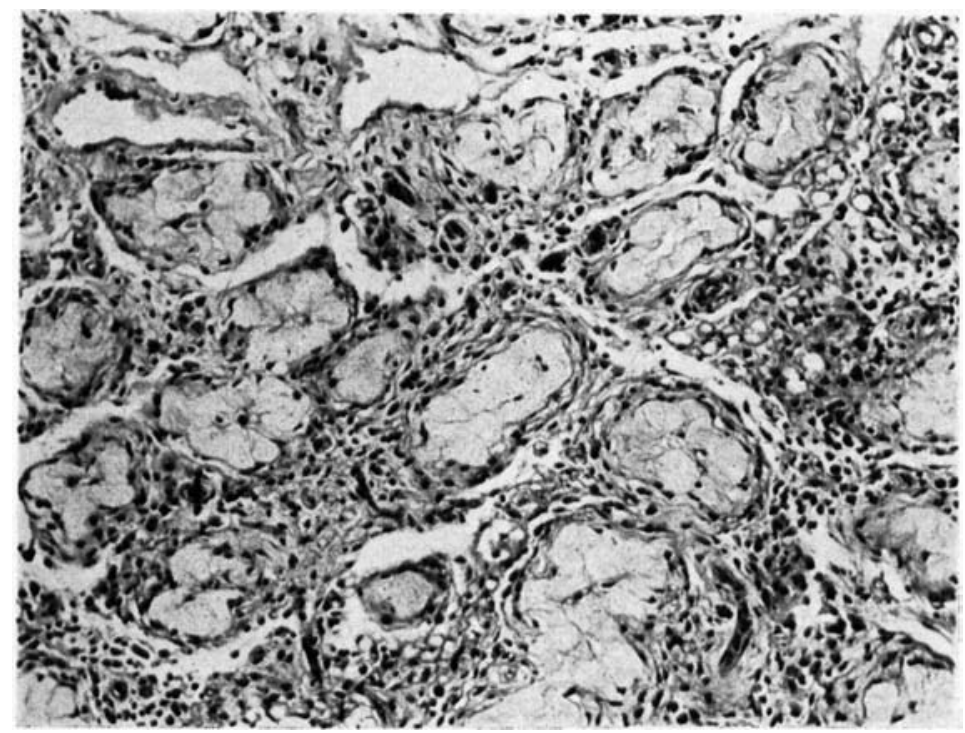

8. Photomicrograph of the testis showing marked hyalinization of the seminiferous tubules. There is also infiltration with lymphocytes, plasma cells, macrophages and small collections of foam cells. $H \& E \times 100$. 
Rodriguez $(1935)^{7}$ of the Philippines, reported a case which he thought presented a typical picture of Lazarine Leprosy. In this case also, the basic histologic picture was that of tuberculoid leprosy. He suggested that Lazarine Leprosy may occur in the Orient also.

Rodriguez and Wade $(1940)^{8}$ reported a case of bullous tuberculoid leprosy. They thought that bullous formations can occur in both lepromatous and tuberculoid leprosy. So Lazarine Leprosy was not a separate type of the disease but was a reaction that occurred in the course of both tuberculoid and lepromatous forms of the disease.

Burks and Brunsting (1941)' reported a case of pemphigoid leprosy. The histopathological examination showed a basic lepromatous picture. There was also congestion of the entire cutis. Acidfast bacilli were seen abundantly throughout the entire section especially in the vessels and nerve bundles. They found acid-fast bacilli in the walls and lumina of blood vessels also.

Latapí and Zamora $(1948)^{4}$ published their study in Lazarine Leprosy. In their histopathological study of the diffuse cutaneous infiltration they found that it was different from the classical nodular type and the acute episodes were essentially dependent on an “acute vascularitis". Latapí had proposed the name "Lucio's Phenemenon" or "Erythema Necrotisans" to this rare manifestation of leprosy.

Pardo Castello and Pineyro (1949) ${ }^{6}$ reviewed the literature on Lazarine Leprosy and concluded that there was no specific form of leprosy that could be called Lazarine, but this term might be used as a synonym for predominantly bullous and necrotic lesions which might occur in either lepromatous or tuberculoid forms. Cochrane ${ }^{2}$ in his text book described Lazarine Leprosy in the words of Latapí and Zamora as a diffuse "lepromatosis". He had looked for this special manifestation in other parts of the. world and had not definitely seen it outside the American continent.

According to $\mathrm{Khanolkar}^{3}$, in the early phase of the disease few types of changes were encountered. Firstly a marked proliferation and mobilization of polyblasts and histiocytes in the dermis and secondly a considerable dilatation, necrosis, and thrombosis of capillaries. At a later phase there was a formation of a soft blister which bursts, leaving an ulcer ringed with a zone of chronic inflammation. This phase is characterised by wide-spread, multiple, necrotising lesions of blood vessels in the skin.

In the case described above, the patient gave a history of having a hypopigmented lesion 16 years previously which later spread all over the body. On examination, a diffuse infiltration of the entire skin of the body was noticed, so a clinical diagnosis of lepromatous leprosy was made. There were also bullous lesions that led to the formation of cutaneous plaques of necrosis and gangrene, followed 
by deep ulceration that extended on to the muscles and tendons, thus causing extensive mutilations of the extremities. The histopathological picture revealed a basic lepromatous pattern. Also, there was inflammation of the vessels of the skin. Some of the vessels showed narrowing of lumina and even thrombosis. Acidfast stain showed numerous bacilli in the corium and in the subcutaneous tissue, especially around the blood vessels, and in their proliferated endothelium.

The clinical and pathological findings show that this is a case which conforms to what has been called "Lazarine Leprosy", a term used to denote leprosy with predominantly bullous and necrotic lesions.

There is a confusion that exists in regard to the correct application of the term "Lazarine Leprosy". The term is used to denote leprosy with predominantly bullous and necrotic lesions. Rodriguez and Wade (1940) commented on the formation of bullae during the course of lepromatous and tuberculoid types of the disease. There seems to be no doubt that there are a number of patients with leprosy who develop bullous lesions. But they can be classified in either one of the two polar forms of the disease. So instead of giving a new name to this particular manifestation in the course of the disease, we might as well call it by a descriptive name "Bullous Reaction" in leprosy.

Bullous reaction in leprosy is not confined only to Mexico and South America. Rodriguez (1955) has reported it in a definite case of tuberculoid leprosy from the Philippines. Our report records bullous formation in a case of lepromatous leprosy from India. The reaction may be seen in countries where lepromatous cases with diffuse infiltration of the skin are commonly met with. In India, lepromatous cases with diffuse infiltration are quite frequently seen, and this case is reported to stimulate others to look carefully for patients with bullous lesions and publish their findings.

\section{Summary}

1. A case of bullous reaction in leprosy is described giving post mortem findings and histopathological observations.

2. A short review of the literature on the subject is given.

3. It is pointed out that Lazarine Leprosy is not a separate entity, but only a phase of the disease in the course of either tuberculoid or lepromatous type of leprosy.

4. It is suggested that bullous reaction in lepromatous leprosy may be found in cases with diffuse infiltration. As such, it may be prevalent in India where this type of case is frequently seen. 


\section{References}

1. Burks, J. W., and Brunsting, L. A., "Lazarine Leprosy-Report of a Case". Proceedings of Mayo Clinic (1941) 16, 488.

2. Cochrane, R. G., "Leprosy in Theory and Practice". John Wright \& Sons, Ltd. (Bristol 1959).

3. Khanolkar, V. R., "Leprosy in Theory and Practice", edited by Cochrane, R. G., John Wright \& Sons Ltd. (Bristol 1959).

4. Latapi, F., and Zamora, A. C., "The Spotted Leprosy of Lucio. An Introduction to its Clinical and Histological Study" (1948) Int. J. Leprosy, 16, 421.

5. Pardo Castello, V., and Caballero, G. M., "Lazarine Leprosy, a Peculiar Monosymptomatic Form of Leprosy" (1931) Arch. Dermat. and Syphilog., 23, 1.

6. Pardo Castello, V., and Pineyro, R., "Lazarine Leprosy-Its Position in the Present Classification of Leprosy" (1949) Int. J. Leprosy, 17, 65.

7. Rodriguez, J. N., "Lazarine Leprosy" (1935) Leprosy in India, 7, 152.

8. RoDriguez, J. N., and WADE, H. W., "Bullous Tuberculoid LeprosyReport of a Case with Discussion of 'Lazarine Leprosy" (1940) Inter. J. Leprosy', 8, 333. 